\title{
MAESTRÍA EN EDUCACIÓN RURAL CENTROAMERICANA: HACIA UN MODELO PEDAGÓGICO FLEXIBLE
}

\author{
Vivian Carvajal Jiménez ${ }^{1}$ \\ Académica de la División de Educación Rural, CIDE-Universidad Nacional \\ Heredia, Costa Rica
}

Recibido 11 de setiembre 2007 • Aprobado 20 de noviembre 2007

\begin{abstract}
Resumen: La Maestría en Educación Rural Centroamericana se propone ante la comunidad nacional e internacional, como un posgrado de alto nivel, cuyo objeto es mejorar la calidad de los procesos educativos formales y no formales en la región centroamericana, a través de un modelo pedagógico innovador que recoge la itinerancia por el istmo y la educación presencial y a distancia con componente virtual. Asimismo, sustenta su práctica en el desarrollo de un modelo flexible, en la inclusividad y en una propuesta metodológica y evaluativa diferenciadas, que recuperan los saberes construidos en cada país centroamericano alrededor del tema de la educación rural.
\end{abstract}

Palabras clave: Modelo pedagógico, educación a distancia e itinerancia, autogestión, componente virtual.

\begin{abstract}
Program in Rural Education" is presented to the national and international community as a high level graduate program, whose purpose is to improve the quality of formal and informal educational processes within the area through an innovative pedagogical model that includes itinerancy along the isthmus, as well as in site and distance learning with a virtual component. Furthermore, this program bases its practice on the development of a flexible model, on inclusiveness and also on a differentiated methodological and evaluative proposal that recovers the knowledge produced in every country around de issue of rural education.
\end{abstract}

Key words: Pedagogical model, distance learning and itinerancy, virtual component.

Hace algunos años la División de Educación Rural se comprometió con la idea de desarrollar un posgrado de alto de nivel que estuviera dirigido hacia el mejoramiento de la calidad educativa rural, formal y no formal, en el istmo centroamericano. Dicha iniciativa encuentra sustento en lo que menciona el informe del estudio exploratorio "Un acercamiento a la Educación General Básica en las zonas rurales de seis países centroamericanos" (UNA/CIDE/DER, 2003), respecto de mejorar la calidad de la educación como el principal desafío al que se enfrenta la región centroamericana en la primera década del siglo XXI.

Coordinadora de la Maestría en Educación Rural Centroamericana. Académica de la División de Educación Rural. UNA. Correo electrónico: vivian.carvajal@gmail.com 
Entre sus hallazgos, el estudio expone que en América Central existen más de 40.000 escuelas primarias con un porcentaje elevado de centros rurales unidocentes y multigrado (en Guatemala, Costa Rica y Nicaragua, alrededor del 50\% del total de las escuelas primarias se ubican dentro de esta categoría). Además, en el nivel universitario, con excepción de una universidad costarricense, los planes de formación dirigidos a las y los docentes rurales son prácticamente inexistentes.

Si se trata de la calidad de la educación formal (en los niveles preescolar, primaria y secundaria), en América Central se han identificado problemas comunes como la falta de articulación entre la educación y el contexto social y productivo de los participantes; la débil atención a los grupos de alta vulnerabilidad a la pobreza y la marginación; la supervivencia de un enfoque educativo que privilegia la memorización; la carencia de material didáctico adecuado a las necesidades de los alumnos; el deterioro y la falta de una infraestructura apropiada para el desarrollo de efectivos procesos de enseñanza y de aprendizaje; las deficiencias respecto de la operacionalización del concepto de multiculturalidad; la falta de disponibilidad de educadores preparados y la escasez de programas de seguimiento y capacitación permanente.

Además, en términos generales, los componentes curriculares presentan limitaciones en cuanto a contextualización, coherencia entre sus partes, integralidad de contenidos y estructura.

Por otro lado, en la región existe una amplia y muy diversa oferta de educación no formal que, dicho sea de paso, suele ser ignorada o minimizada por la educación formal; aunque muchos centros funcionan como alternativa o complemento de la institucionalidad y la academia.

Con base en lo anterior, era evidente que, se requería de un proyecto específico que contemplara la complejidad que encierra la realidad centroamericana, porque a pesar de su cercanía geográfica, existe una diversidad cultural que es necesario tomar en consideración para lograr satisfactorios resultados educativos, proyectivos y desarrollistas.

En otras palabras, el mejoramiento de la calidad de la educación rural es fundamental para el desarrollo de la región centroamericana. No obstante, se trata de un proceso que demanda el compromiso de diversos actores individuales y colectivos; disponibilidad de los múltiples recursos que cada país posee; y voluntad política de los gobiernos y de la sociedad en general. Es un proceso social que debe ser promovido desde diversas instancias que mancomunen esfuerzos para romper con las diferencias estructurales entre el medio rural y el medio urbano; dado que, durante siglos, la pobreza se ha asociado a la ruralidad, lo que representa una paradoja, pues la tierra por sí sola es valiosa, bien sea por ser considerada como moneda de cambio o porque en y de ella se puede cultivar, construir, alimentar ganado, rentar, vivir.

Las zonas rurales de casi todos los países de Centroamérica presentan los índices de menor desarrollo humano. La urgencia de intervenir en la educación rural es aún más apremiante cuando todos los indicadores y marcadores económicos muestran claramente importantes diferencias de desarrollo social en las áreas rurales con respecto de las urbanas. En otras palabras, la pobreza se concentra en las zonas rurales, y sigue teniendo rostro de campesino, de pescador, de indígena, de inmigrante, de mujer, de niño.

Lo que no se explica, es que la ruralidad tiene una enorme barrera que la hace vulnerable, y en eso consiste precisamente su debilidad y su esencia: está aislada del lugar donde se produce, se construye y reconstruye permanentemente el conocimiento oficialmente reconocido.

En síntesis, la ruralidad está incomunicada, porque se ubica lejos del sitio donde se desarrollan procesos de producción y reproducción del conocimiento oficializado. Las universidades -generadoras de conocimiento y desarrollo- se encuentran en los centros urbanos, muy cerca de los núcleos de poder, y muy lejos de las zonas rurales dispersas. Por ello, la División de Educación Rural ha entendido que su compromiso consiste en acercarse a las comunidades rurales y aproximar 
a sus académicos a estas; así explica su itinerancia, su afán por abrir espacios para investigar, innovar, adecuar, actualizar y hacer pertinentes la educación y los programas que administra a la realidad y al acontecer de lo rural centroamericano.

De esta forma, con la idea de visibilizar la situación y riqueza de las comunidades rurales centroamericanas y generar encuentros, proyectos, discusiones y trabajos que apoyaran el desarrollo rural desde la educación formal y no formal del istmo, y aprovechando la experiencia que ha acumulado la División de Educación Rural durante tres décadas; en febrero de 2006 dio inicio la primera promoción de la Maestría en Educación Rural Centroamericana (MERC), cuyo trabajo culminó hacia finales de agosto del 2007.

La MERC ha innovado metodológicamente por componentes como la itinerancia por Centroamérica, la participación de estudiantes de todo el istmo y la entrega de la docencia a través de medios virtuales; además, porque ha rescatado y valorado el saber construido en las comunidades rurales centroamericanas, tanto como el conocimiento conceptual y académico que se cosecha en la región.

Asimismo, mediante un programa como este, se ha promovido el sentido de unidad centroamericana, y se han creado vínculos que sin duda favorecerán el trabajo participativo en la región a través del encuentro, las giras por diferentes países, la lectura y análisis de textos y realidades, y el desarrollo individual de un proyecto dirigido hacia las comunidades rurales del país y localidad de origen de cada participante. Paralelamente, la construcción y deconstrucción cognitiva y afectiva, producto del análisis de experiencias y el enfrentamiento entre la teoría y la práctica, contribuyen con el cuestionamiento de estereotipos, prejuicios y toda posición que atente contra la equidad y los derechos humanos.

Nuestro programa surge como el resultado de un cuidadoso estudio llevado a cabo por el equipo de la División de Educación Rural durante varios años. Investigaciones preliminares arrojaron resultados en el sentido de que no existía en la región ninguna propuesta que tuviera como objeto de estudio la educación formal y no formal en el ámbito rural, mucho menos, con una visión regionalista.

En el año 2001, en el marco del proyecto "Fortalecimiento de las escuelas rurales de Costa Rica y Centro América", fueron estudiadas y evaluadas diferentes experiencias en educación en los distintos países centroamericanos. Producto de esta investigación, se comprenden las fortalezas que cada país puede aportar para desarrollar los distintos módulos de la Maestría, incorporando además la itinerancia centroamericana como un componente enriquecedor que posibilita construir una visión panorámica, integral y holística de la región.

Así, la experiencia de la División de Educación Rural en el desarrollo de sus actividades académicas, con la modalidad presencial y a distancia, permitió que se asumiera la tarea de combinar en la Maestría la modalidad presencial, virtual y a distancia mediante la tutoría, pues la experiencia ya consolidada en la DER se enriqueció con la capacitación del equipo en el manejo de las nuevas tecnologías de la información y de la comunicación, el trabajo con la plataforma Moddle y otros mecanismos comunicativos.

El programa de la maestría se constituye por el conjunto de procesos educativos formales y no formales que tienen cabida en el área rural centroamericana, enfatizando los procesos de enseñanza y aprendizaje individuales y grupales, que se definen como dinámicos y cambiantes, inscritos en una realidad social igualmente dinámica, y que señala a los actores sociales implicados como protagonistas de estos procesos.

Como se puede deducir, esta es una importante oportunidad para abrir espacios académicos de investigación y socialización de los hallazgos del trabajo con comunidades rurales, uno de los 
obstáculos más grandes a los cuales se enfrenta la educación rural, debido a las condiciones de aislamiento en que, muchas veces, se encuentran estas regiones.

Por otro lado, la misma composición del grupo de estudiantes de la maestría conforma por sí sola una red. En otras palabras, tener en un aula o grupo a personas de toda Centroamérica, en medio de espacios para compartir, construir, discutir, trabajar, comunicar, etcétera; facilita la construcción de nuevos lazos de entendimiento, de miradas desde la centroamericanidad, de redes afectivas que revierten en nuevas conductas sociales y en convivencias nuevas.

Por eso, la población de la Maestría se compone de profesionales y docentes rurales, asesores, autoridades de ministerios, promotores de ONGs, coordinadores de educación de gobiernos locales, personal responsable de procesos y programas educativos, y todas las personas que reúnan los requisitos y que manifiesten interés por conocer experiencias similares y diversas que se desarrollan en la región y que tengan el compromiso de promover y facilitar procesos educativos formales y no formales, que lleven a mejorar la calidad de vida de los habitantes de las zonas rurales, y de desarrollar las condiciones personales que permitan una mejor calidad de vida, así como las condiciones ambientales para que estos mejoramientos sean duraderos.

El programa de la MERC hace énfasis en el desarrollo humano sostenible, en la equidad de género y en la promoción de una metodología participativa. Aunque el posgrado se desarrolla por primera vez entre 2006-2007, su pertinencia y actualización se han evaluado en la medida en que se trabajan los módulos, ya que tanto en la programación presencial como en la etapa a distancia, se aplican instrumentos y se separan espacios para indagar estos aspectos. Los resultados han sido altamente satisfactorios. Sin embargo, existe mucha apertura y claridad sobre la necesidad de mantener atenta la mirada a la actualización de los temas, debido a la velocidad -casi alucinante- del cambio de los escenarios, los actores y los acontecimientos de la región centroamericana.

Por otro lado, la incursión del componente virtual como complemento del trabajo a distancia, suministra un valor agregado en lo que refiere a los conocimientos construidos por tutores y estudiantes respecto de las estrategias comunicativas, el seguimiento, la flexibilidad evaluativa y la actualización del personal académico de la DER.

Al respecto, vale la pena destacar el hecho de que la educación a distancia, una modalidad que permite a diferentes grupos el acceso a la educación y a formas de aprender que antes estaban limitadas a ciertos contextos o circunstancias, favoreció la incursión de modelos y la creación de estrategias que, de forma innovadora, promueven el autoaprendizaje y la independencia del estudiante en los procesos de construcción y relaciones de conocimiento; y a su vez, facilitan la apropiación de técnicas, ritmos y horas dedicadas a la tarea de aprender, y por supuesto, de tutorear.

Entonces, la educación flexible, vinculada en este caso con el desarrollo de tareas a distancia y con la implementación de un programa educativo bimodal, supone “... una nueva concepción, que independientemente de si la enseñanza es presencial o a distancia, proporciona al alumno una variedad de medios y la posibilidad de tomar decisiones sobre el aprendizaje..." (Salinas, 2005, 3).

En esta línea, Moran y Myrlinger (2000) definen el ideal de aprendizaje flexible como un enfoque de enseñanza y aprendizaje centrado en el alumno, con amplios grados de libertad en el tiempo, el lugar, y los métodos de enseñanza y aprendizaje; además, con un uso adecuado de las tecnologías apropiadas a la población participante, en un entorno en red.

Por su parte, Wedemeyer (1981) citado por Salinas (2006), se basa en un ideal social democrático y en una filosofía educativa liberal que retoma la igualdad de oportunidades para acceder a la educación, de manera que se superen las limitaciones geográficas, económicas y sociales. En cambio, Holmberg (citado por Salinas, 2006) se refiere al estudio a distancia como un 
autoaprendizaje, y añade que el alumno cuenta con el soporte de la institución que desarrolla el curso y con la interacción con el tutor y otros miembros de la organización. Esta concepción visualiza los procesos de aprendizaje a través del diálogo y la interacción entre estudiantes y docentes; pero es el participante quien, de manera autónoma, elige, elabora, selecciona, interviene, propone o pospone.

Otro aspecto vital en la definición del currículo que se selecciona e implementa en estos casos, es su pertinencia contextual. Sobre este asunto, Peralta (1996) señala que la elección apropiada del currículo, en cuanto a su adecuación cultural y contextual, es indispensable para que se desarrollen estudiantes potencialmente activos, interesados y responsables. Si el currículo es apropiado y pertinente en ambos sentidos, la mediación y los contenidos probablemente serán significativos para los estudiantes-participantes, y se impulsará el desarrollo de valores útiles y prácticos en cada sociedad. Además, un currículo culturalmente pertinente responde a las necesidades de los alumnos, y no a las demandas de los grupos dominantes, del profesor o de la institución facilitadora.

En esta dirección, los programas de aprendizaje flexible deben comprender -y actuar en consecuencia- que sus usuarios “ ...no son los mismos (no presentan las mismas necesidades de aprendizaje, las mismas motivaciones, la misma independencia, situaciones laborales y profesionales, las mismas condiciones y disponibilidades, etcétera), y que ... no pretenden los mismos aprendizajes, los que aprenden desde el hogar que los que lo hacen desde el puesto de trabajo o en un centro educativo convencional ..." (Salinas, 2003, p. 73).

Con la MERC, se busca una suerte de modelo hermenéutico-reflexivo que orienta al participante, sea docente o alumno, comprometido con sólidos valores (no neutro) y con competencias polivalentes. Se trata de un individuo abierto, capaz de:

- Partir de la practica como eje estructurante, tanto áulica, institucional y comunitaria, como social.

- $\quad$ Problematizar, explicitar y debatir desde la bibliografía escolar previa hasta las situaciones cotidianas, las creencias, las rutinas, los estereotipos, las resistencias, los supuestos, las relaciones sociales, los proyectos; así como los contenidos, los métodos y las técnicas.

- Reconstruir la unidad y complejidad de la propia experiencia docente contextualizada, con sus implicaciones emocionales, intelectuales, relacionales y prospectivas.

- Compartir la reflexión personal critica en ámbitos grupales contenedores, con coordinación operativa, y posibilitar así cambios actitudinales.

- $\quad$ Propiciar imprescindibles espacios de investigación cualitativa con participación protagónica de los actores comunitarios, utilizando métodos diversos. Entre ellos: cartas, bitácoras personales, diarios de campos, testimonios orales, historias de vida, casos reales o simulados, documentales, dramatizaciones ...

- $\quad$ Leer, tratando de interpretar los signos de los tiempos, las necesidades y potencialidades del entorno rural.

- Constituirse en referente teórico-metodológico en lo relativo a la pedagogía rural.

Por otro lado, existen varios modelos pedagógico-comunicativos que determinan la manera en que se desarrollan las relaciones de enseñanza y de aprendizaje en el aula, sea física o virtual.

Paran nuestro caso, es el Modelo pedagógico social cognitivo el que se sugiere como orientador de una práctica educativa flexible. Dicha corriente parte de la premisa del desarrollo máximo y multifacético de las capacidades e intereses del estudiante. Acepta la influencia social y colectiva del entorno, y reconoce la estrecha vinculación entre escuela y comunidad. El método se basa en el estudio de problemas y situaciones tomados del contexto de los estudiantes, lo que ofrece la 
motivación de plantear soluciones concretas e inmediatas a casos cercanos que así lo requieran. Le otorga importancia a la capacidad de observación y a la participación constante. La evaluación es dinámica e interactiva, lo mismo que la comunicación entre todos los involucrados. Por ello, la MERC, durante sus encuentros presenciales, destacó como facilitadores a quienes hubiesen cosechado experiencias valiosas en las áreas de la educación no formal, la participación comunitaria, la multiculturalidad, la educación para el trabajo, la implementación de tecnologías en zonas rurales, la escuela unidocente, la organización comunitaria, la gestión de proyectos y la pedagogía rural, entre otros; independientemente de si dicho conocimiento está adscrito a la Academia, la universidad, el ministerio de educación, una cooperativa, una junta de desarrollo, un grupo de mujeres, una escuela comunitaria, una ONG o cualquier otro tipo de organización.

Por lo tanto, asegurar la inclusividad de la propuesta no sólo tiene que ver con la selección de determinado currículo, la planificación según X modelo pedagógico, comunicativo, evaluativo y didáctico, la presentación de los materiales o el papel del profesor; sino con la participación genuina y significativa de los actores, los expertos en la temática; y además, con la selección tecnológica precisa, diseñada para la población específica con la que va a trabajarse, con la exposición de materiales debidamente contextualizados y diseñados a partir de los recursos, intereses, necesidades y potencialidades de sus usuarios.

Otro elemento vital es el principio de que la educación debe ir a la gente (Moran y Myrlinger, 2000). Un aspecto atractivo en cualquier proyecto que implique la itinerancia o la educación a distancia, es la oportunidad que abre en cuanto a la cercanía, el tiempo y el espacio: personas de muy distintas ocupaciones, con los horarios más diversos, en variedad de circunstancias económicas, físicas y geográficas, pueden conformar un equipo, estudiar, actualizarse, capacitarse e investigar.

Además, gente con la más amplia gama de habilidades, destrezas, experiencias e inquietudes puede interactuar, compartir, inquirir, cuestionar y realimentar el trabajo de otros, sin que ninguno de ellos deba trasladarse cotidianamente desde su comunidad o trabajo, permitiendo entonces que, a un costo muy bajo, se tenga acceso no sólo al campus en cuestión, sino a expertos de diferentes países y universidades; y a la posibilidad de compartir y construir -junto a gente de todas partessus propias posiciones y conocimientos.

Otra fortaleza de la MERC, es el haber reconocido la importancia del contacto y la relación social, así como de la interacción individual, con los otros participantes, con el material de apoyo y con las herramientas didácticas.

Los seres humanos somos seres sociales, y en la educación a distancia, es imprescindible explotar el recurso comunicativo y las oportunidades de intercambio que la tecnología ofrece. En estos medios, es frecuente el acercamiento y el planteamiento de dudas y comentarios, dado que el estudiante no se siente intimidado ni presionado por el grupo o la "mirada" del profesor. Además, ofrece la posibilidad de plantear con calma las inquietudes, y pensar y repasar las intervenciones antes de hacerlas públicas, o bien, luego de haber digerido lo vivenciado durante a fase presencial.

No obstante, también es cierto que en la distancia se pierden los elementos de contacto que facilitan el desarrollo de una lección, la explicación de un tema o la lluvia -simultánea- de ideas alrededor de un tema. No se cuenta con la gestualidad ni la gesticulación, con la pizarra ni los ejemplos que la pericia del docente puede sacar en el momento apropiado, con las anécdotas ni las ilustraciones que saltan al caso. Sin embargo, sí se cuenta con la tutoría constante del facilitador, y con la comunicación continua con los otros participantes, lo cual favorece el esclarecimiento de dudas y estrecha la relación con el grupo. Por ello, la combinación de las modalidades -itinerante, presencial y a distancia- favorece la satisfacción de diversidad de estilos de aprendizaje y de condiciones locales o personales. 
Asimismo, el intercambio individual, progresivo y continuo que cada estudiante realiza con los materiales del curso y con las herramientas de trabajo disponibles, es sumamente rico, y varía de un individuo a otro, tanto en tiempos como en ritmos e intereses.

En esta línea, Bates (1991) citado por Salinas (2006) señalaba dos tipos muy diferentes de interactividad en el aprendizaje: social e individual. La interacción social entre los alumnos y el profesor necesita balancearse mediante la interacción individual del alumno con los recursos de aprendizaje, incluyendo libros de texto, guías de estudio, vídeos y programas de aprendizaje asistidos por computadora. La enseñanza tradicional pone el énfasis en la interacción social, en tanto que Modelo multimedia se esfuerza en proporcionar calidad a la interacción individual del alumno con los materiales.

Por otro lado, debido a que los participantes de este tipo de posgrado se ubican en sitios geográficos diversos, tienen horarios distintos y ocupaciones varias; la libertad en el tiempo, horario y lugar de trabajo es absoluta: desde dónde pueda y desde dónde le resulte más cómodo, a la hora en que mejor se adapte, y al ritmo que su estilo de aprendizaje avance, así irá profundizando y desarrollando sus tareas. En este tipo de proyectos, además, se suele delegar una amplitud de responsabilidad y libertad en el alumno, que no es usual en otro tipo de cursos: en muchas ocasiones el estudiante puede proponer, designar fechas de entrega, diseñar y ofrecer correcciones.

De esta forma, siguiendo principios cognoscitivistas, nuestra propuesta se centra en el estudiante: qué puede lograr, cómo soluciona los problemas, qué propuestas genera, cómo percibe el entorno, qué le interesa. Así, tanto las estrategias de mediación pedagógica como la propuesta evaluativa, procura el desarrollo de destrezas particulares, el trabajo metacognitivo y el protagonismo del estudiante, en tanto que el tutor o facilitador es un guía, un motivador y un "recurso" que, por su experticia, el propio alumno puede explotar.

Si para el estudiante de este tipo de maestría existen tantos alicientes y protagonismo; para el facilitador no es menos atractivo el reto: como tutor de un proyecto que desarrolle la educación itinerante, presencial y a distancia con apoyo de las TIC, su papel de profesor evoluciona de supervisor a dialogador e investigador. Se trata de un orientador de propuestas, de un incentivador intelectual, de un promotor del debate y de un "cocinero" de experiencias.

Para Salinas (2005) “...el rol del profesor cambia de la transmisión del conocimiento a los alumnos a ser facilitador en la construcción del propio conocimiento por parte de estos. Se trata de una visión de la enseñanza en la que el alumno es el centro o foco de atención y en el que el profesor juega, paradójicamente, un papel decisivo. Adoptar un enfoque de enseñanza centrada en el alumno significa atender cuidadosamente a aquellas actitudes, políticas y prácticas que pueden ampliar o disminuir la distancia de los alumnos distantes. El profesor actúa como persona y después como experto en contenido. Promueve en el alumno el crecimiento personal y enfatiza la facilitación del aprendizaje antes que la transmisión de información” (p. 15).

Por si esto fuera poco, desde cualquier otra perspectiva, la oportunidad de atender simultáneamente y en un mismo curso, a estudiantes de diferentes países, edades, ocupaciones e intereses, sería financieramente imposible. La modalidad por la que la DER ha optado para este programa realmente permite reducir enormes costos de traslado, papel, impresión de documentos y trámites administrativos. De igual manera, a muy bajo costo, divulga su quehacer y obtiene insumos para su trabajo.

Como ya se ha mencionado, por tratarse de un programa con carácter regional, y también, por implicar el trabajo a distancia en línea, cualquier interesado, en cualquier parte del mundo, puede acceder a la información, participar de un modulo o suministrar datos o colaboraciones 
que enriquezcan la tarea del curso en general, o de un estudiante en particular. Asimismo, se divulgan los logros, procesos, proyectos, publicaciones y alcances de la entidad patrocinadora de la propuesta.

Finalmente, por su alcance, lo reducido de sus costos, y la libertad en cuanto a tiempos, espacio y ritmo de aprendizaje, personas que pertenecen a grupos tradicionalmente excluidos (por su etnia, su condición económica, su procedencia geográfica, su ritmo de aprendizaje o alguna discapacidad), pueden acercarse al programa que aquí nos ocupa. No obstante, un factor social que es particularmente significativo para los programas educativos a distancia, es el de presencia social, entendido como el grado en que una persona se siente socialmente presente en una situación mediada. La idea es que la presencia social en la MERC, sea inherente al posgrado mismo, y que las tecnologías les ofrezcan a los participantes una variación de grados de dicha presencia.

Cuando se trata de propuestas metodológicas que involucren el trabajo a distancia, y la disciplina, iniciativa y responsabilidad que demandan los cursos con esas particularidades, es necesario priorizar en los ejes que deben orientar la práctica pedagógica de manera global; esto es, la filosofía o corriente educativa que inspire y origine el currículo, las actividades, la evaluación, los objetivos, las competencias por alcanzar, la dinámica de trabajo que permitirá la realización de la propuesta y, desde luego, la caracterización de los sujetos que resultarán actores de tal programación.

En el caso específico de la MERC, existe toda una serie de dimensiones que deben atenderse de manera apropiada. Entre ellas, quizá la más importante, sea la que implica al estudiante de la modalidad; pues comprendido desde los enfoques cognoscitivista y humanista, es el sujeto que construye, elabora, aprehende, investiga, evalúa, escoge y elabora; quien debe constituirse en prioridad del proyecto, en el entendido de satisfacer sus necesidades, permitiéndole, eso sí, desarrollar su potencial y ahondar en las áreas que le resulten más significativas o interesantes.

El participante de un programa educativo a bimodal debe ser un individuo con alta capacidad de autoaprendizaje (y por ello, con todas las habilidades que esa condición implica) y un elevado nivel de compromiso hacia la tarea que está emprendiendo, y en este caso, hacia las poblaciones rurales de la región.

¿Y qué significa poseer capacidad de autoaprendizaje? Se trata de un proceso al que se somete un individuo con el interés de aprender alguna cuestión teórica o técnica, con la conciencia de que deberá lograrlo poniendo su máximo empeño en ello y de que lo hará por sus propios medios, en tiempos que él decida. El autoaprendizaje es un mecanismo intelectual que funciona con base en el criterio prueba-error, en que la persona tiende a digerir información referente a procesos individuales.

En otras palabras, el autoaprendizaje está ligado con toda una serie de habilidades cognitivas que tienen que ver con el intelecto, pero que además, pueden ejercitarse e incluso, aprenderse mediante ciertas técnicas y prácticas que deben volverse parte de la cotidianidad, si es que se trata de aprendizaje permanente. Esto, por supuesto, no puede desarrollarse sin el interés ni la motivación intrínseca del participante, pues son propias inquietudes y experiencias las que deben orientar su trabajo: qué investigar, qué desea conocer, en qué áreas quisiera profundizar, en cuáles aspectos puede aportarles a los otros participantes, de qué manera involucrar a otros...

Es importante gestionar planes organizativos porque, aunque el estudiante puede trabajar cuando tenga tiempo o energía, debe planificar cuidadosamente un calendario de trabajo o un compromiso horario que le permita abarcar el material y ejecutar las tareas semanalmente. Por supuesto, esto requiere de disciplina, responsabilidad y compromiso.

Sin embargo, la motivación extrínseca que el programa en cuestión estimula en el estudiantado es también muy significativa; de ahí que los contenidos, actividades y materiales que se abarcan, 
responden a un modelo educativo autogestionario, promotor de crítica y gestor de interrogantes investigativas que obligan al cuestionamiento, la crítica y la reflexión.

Por otro lado, cuando se trata de un programa que, como este, implica componentes virtuales, el perfil del estudiantado debe incluir la incorporación de la cultura informática básica, pero especialmente, disposición para adiestrarse en el manejo de estas herramientas y de la misma manera, suplir cualquier otra necesidad referida a las destrezas mínimas que el programa demanda: capacidad de redacción, lectura analítica, etc.

En cuanto al recurso humano que se desempeña en la MERC, es imprescindible la capacitación y la experiencia que permitan hacer frente al desarrollo de los módulos. Por un lado, en el aspecto disciplinar, la experticia, iniciativa y actualización de los tutores y facilitadores resulta absolutamente necesaria; y por otro, en el manejo de situaciones relacionadas con la virtualidad, pues esto reduce la necesidad de personal adicional, ajeno al área de conocimiento que se desarrolla, dedicada a cuestiones ligadas con la presentación de materiales y actividades que deben incrustarse en una plataforma. Para que el desempeño del equipo de trabajo sea exitoso, la capacitación debe ser constante, y la labor investigativa ha de constituirse en eje transversal de toda la práctica.

Por su parte, los aspectos pedagógicos relacionados con la virtualidad del programa, así como con el aprovechamiento del componente itinerante, tienen que ser trabajados desde el interior mismo de este, y deben mostrar total coherencia con el modelo conductor de la propuesta; a saber, características y objetivos de las experiencias de aprendizaje, forma en que la evaluación es concebida, herramientas informáticas que se incorporarán al plan de trabajo, estrategias para promover la interacción entre los participantes y entre estos y sus tutores, técnicas que favorezcan la investigación y la autogestión, características del material de apoyo, etc. Desde luego, este conjunto debe permanecer bajo constante revisión y ser objeto de discusiones y valoraciones que permitan realimentar cualquier propuesta, actividad o método.

Entre las fortalezas de la MERC destacan el alto grado de compromiso del equipo de trabajo y de la Dirección de la Unidad Académica a la que se encuentra adscrito el posgrado, lo cual, desde el punto de vista pedagógico, es un éxito. Igualmente, contar con un programa que integra a cuatro docentes como equipo, de manera interdisciplinaria, y el acompañamiento dado a los estudiantes y a los encargados del diseño de cada módulo, fueron aspectos clave que ayudaron a tener un bajo índice de deserción, mantener la motivación de los participantes, construir procesos pedagógicos innovadores y desarrollar un alto nivel de compromiso e identificación con el posgrado, tanto entre estudiantes como entre docentes y administrativos.

En síntesis, la MERC prioriza la afectividad, la solidaridad, el compromiso, el respeto hacia la naturaleza, la equidad de género y étnica, el liderazgo, el desarrollo de habilidades que potencien el uso de recursos tecnológicos de manera sostenible, la participación local y la responsabilidad social, con el fin de empoderar a los participantes, a los residentes o trabajadores de zonas rurales y a sus respectivas comunidades. Su gestión se caracteriza por el trabajo participativo, interdisciplinario y transdisciplinario, la coordinación con unidades académicas y sedes regionales de la UNA, con universidades centroamericanas, asociaciones, instituciones y con organizaciones gubernamentales y no gubernamentales de toda la región.

Hemos rescatado durante cinco módulos el quehacer académico y comunitario que ilustra las fortalezas desarrolladas por nuestros países través de experiencias, conferencistas, conversatorios con participación de expertos de muy diversas regiones, documentales, visitas a centros de estudios, escuelas, universidades, asociaciones de desarrollo, cooperativas, escuelas normales, ministerios, organizaciones, proyectos; en fin, una variedad de situaciones de aprendizaje que al mismo tiempo 
que nos llevaban a reconocer las fortalezas y necesidades educativas formales y no formales de nuestros países, nos permitieron conocer el panorama centroamericano en múltiples dimensiones, más allá de los prejuicios, de lo que los noticiosos suelen divulgar, o de lo que el turismo promueve; y que a la vez, nos posibilitaron la creación de vínculos que favorecen nuestro trabajo profesional.

A veces, nuestro posgrado se llamó educación no formal, a veces, se llamó educación para el trabajo; a veces, era todo multicultural o participación; otras sólo pudimos ver los desafíos, o los logros; en ocasiones, nos quedamos impactados por el trabajo realizado, por la pobreza que es ignorada, por los estragos del conflicto armado, por la fortaleza de nuestros hermanos centroamericanos.

Si hoy pudiéramos renombrar la MERC, deberíamos llamarla Compromiso, porque luego de pasar revista por la realidad rural centroamericana, ninguno de los involucrados es la misma persona; porque a pesar del esfuerzo maratónico, organizativo, económico y de seguimiento que implicó para todos los que de una u otra manera participamos en esta primera promoción, estamos más convencidos que nunca de la necesidad de posgrados que volteen su mirada hacia las necesidades de quienes por tradición han sido "olvidados"; los frutos arrojados ya se dejan ver, y es evidente que el impacto en nuestros países se hará sentir en beneficio del mejoramiento de la calidad de vida de nuestras comunidades.

\section{REFERENCIAS}

Moran, L. y Myringer, B. (1999). Flexible learning and university change [Entornos virtuales y formación flexible]. En H. Keith (Ed.), Higher EducationThrough Open and Distance Learning (pp. 52-72). Londres: Routledge.

Peralta, V. (1996). Curriculos educacionales en América Latina. Buenos Aires: Editorial Andrés Bello.

Salinas, J. (2003). Entornos virtuales y formación flexible. Tecnología en marcha, 17 (3) especial, 69-80.

Salinas, J. (2005). Cambios metodológicos en las TIC. Estrategias didácticas y entornos virtuales de enseñanza-aprendizaje. Palma de Mallorca, España: UIB.

Salinas, J. (2006). La formación flexible entre la enseñanza presencial y la educación a distancia: Modelos y experiencias. Palma de Mallorca, España: UIB.

UNA-CIDE-DER. (2003). Mejoramiento cualitativo de la Educación Básica en las comunidades rurales de Centroamérica: aportes desde la Educación Superior. Documento de proyecto. 